\title{
Axially Symmetric Vibrations of Composite Poroelastic Spherical Shell
}

\author{
Rajitha Gurijala and Malla Reddy Perati \\ Department of Mathematics, Kakatiya University, Andhra Pradesh 506009, Warangal, India \\ Correspondence should be addressed to Malla Reddy Perati; mperati@yahoo.com
}

Received 26 December 2013; Accepted 9 March 2014; Published 28 April 2014

Academic Editor: Z.X. Guo

Copyright (C) 2014 R. Gurijala and M. R. Perati. This is an open access article distributed under the Creative Commons Attribution License, which permits unrestricted use, distribution, and reproduction in any medium, provided the original work is properly cited.

\begin{abstract}
This paper deals with axially symmetric vibrations of composite poroelastic spherical shell consisting of two spherical shells (inner one and outer one), each of which retains its own distinctive properties. The frequency equations for pervious and impervious surfaces are obtained within the framework of Biot's theory of wave propagation in poroelastic solids. Nondimensional frequency against the ratio of outer and inner radii is computed for two types of sandstone spherical shells and the results are presented graphically. From the graphs, nondimensional frequency values are periodic in nature, but in the case of ring modes, frequency values increase with the increase of the ratio. The nondimensional phase velocity as a function of wave number is also computed for two types of sandstone spherical shells and for the spherical bone implanted with titanium. In the case of sandstone shells, the trend is periodic and distinct from the case of bone. In the case of bone, when the wave number lies between 2 and 3 , the phase velocity values are periodic, and when the wave number lies between 0.1 and 1 , the phase velocity values decrease.
\end{abstract}

\section{Introduction}

In day-to-day problems, composite structures play an important role. The term composite is applied to materials that are created by mechanically bonding two or more different elastic materials together. On the other hand, in structural engineering, spherical shell shape trims the internal volume and minimizes the surface area that saves material cost. Spherical shell forms an important class of structural configurations in aerospace as well as ground structures as they offer high strength-to-weight and stiffness-to-weight ratios. Besides some of manmade structures, the skull and the bones at shoulder joint and ankle joint are approximately in the shape of spherical shells. The said composite spherical structures are poroelastic in nature.

Kumar [1] studied the axially symmetric vibrations of fluid-filled spherical shells employing three-dimensional equations of linear elasticity. For torsional vibrations of solid prolate spheroids and thick prolate spheroidal shells, frequency equations and mode shapes are presented in analytic form [2]. Paul [3] studied the radial vibrations of poroelastic spherical shells. Employing Biot's theory [4],
Shah and Tajuddin $[5,6]$ discussed torsional vibrations of poroelastic spheroidal shells and axially symmetric vibrations of fluid-filled poroelastic spherical shell. In the paper in [5], they derived frequency equations for poroelastic thin spherical shell, thick spherical shell, and poroelastic solid sphere and concluded that the frequency is the same for all the three cases. In the paper in [6], radial and rotatory vibrations of fluid-filled and empty poroelastic spherical shells are investigated. Vibration analysis of a poroelastic composite hollow sphere is discussed by Shanker et al. [7]. They derived frequency equations for poroelastic composite hollow sphere and a poroelastic composite hollow sphere with rigid core. Some structures may be far from their center of curvature and thickness might be very small when compared to radii of curvature. In this case, we have ring modes [8]. To the best of authors knowledge, poroelastic composite spherical shell and its ring modes are not yet investigated. Hence the same are warranted. In the present paper, we investigate the axially symmetric (independent of azimuthal coordinate) vibrations of composite poroelastic spherical shell in the framework of Biot's theory. Frequency equations are obtained for both pervious and impervious surfaces. Also, frequency against 
the ratio of outer and inner radii and the phase velocity against the wave number are computed. Comparative study is made between the modes of composite spherical shell and its ring modes.

The rest of the paper is organized as follows. In Section 2, basic governing equations, formulation, and solution of the problem are given. In Section 3, frequency equations are derived for both pervious and impervious surfaces. Particular case is derived in Section 4, while numerical results are presented in Section 5. Finally, conclusion is given in Section 6.

\section{Governing Equations and Solution of the Problem}

The equations of motion of a homogeneous, isotropic poroelastic solid [4] in the presence of dissipation $b$ are

$$
\begin{gathered}
N \nabla^{2} \vec{u}+(A+N) \nabla e+Q \nabla \varepsilon \\
=\frac{\partial^{2}}{\partial t^{2}}\left(\rho_{11} \vec{u}+\rho_{12} \vec{U}\right)+b \frac{\partial}{\partial t}(\vec{u}-\vec{U}), \\
Q \nabla e+R \nabla \varepsilon=\frac{\partial^{2}}{\partial t^{2}}\left(\rho_{12} \vec{u}+\rho_{22} \vec{U}\right)-b \frac{\partial}{\partial t}(\vec{u}-\vec{U}),
\end{gathered}
$$

where $\nabla^{2}$ is the Laplace operator, $\vec{u}(u, v, 0)$ and $\vec{U}(U, V, 0)$ are solid and fluid displacements, $e$ and $\varepsilon$ are the dilatations of solid and fluid, $A, N, Q, R$ are all poroelastic constants, $b$ is the dissipative coefficient, and $\rho_{i j}$ are mass coefficients. The relevant solid stresses $\sigma_{i j}$ and fluid pressure $s$ are

$$
\begin{gathered}
\sigma_{i j}=2 N e_{i j}+(A e+Q \varepsilon) \delta_{i j} \quad(i, j=1,2,3), \\
s=Q e+R \varepsilon .
\end{gathered}
$$

In (2), $\delta_{i j}$ is the well-known Kronecker delta function.

Let $(r, \theta, \phi)$ be the spherical polar coordinates. Consider a composite isotropic poroelastic spherical shell with outer and inner radii $r_{2}$ and $r_{1}$, respectively, made up of two different materials and the inner one is solid spherical shell whereas the outer one is a thick walled hollow spherical shell having thickness $h=\left(r_{2}-r_{1}>0\right)$. Poroelastic constants of outer shell and inner shell are ${ }_{2} P,{ }_{2} N,{ }_{2} Q,{ }_{2} R$ and ${ }_{1} P,{ }_{1} N,{ }_{1} Q,{ }_{1} R$, respectively. We introduce the displacement potentials $\phi$ 's and $\psi$ 's which are the functions of $r, \theta$, and $t$ as follows:

$$
\begin{gathered}
u=\frac{\partial \phi_{1}}{\partial r}+\frac{1}{r} \frac{\partial^{2} \psi_{1}}{\partial \theta^{2}}+\frac{\cot \theta}{r} \frac{\partial \psi_{1}}{\partial \theta}, \\
v=\frac{1}{r} \frac{\partial \phi_{1}}{\partial \theta}-\frac{\partial^{2} \psi_{1}}{\partial r \partial \theta}-\frac{1}{r} \frac{\partial \psi_{1}}{\partial \theta}, \\
U=\frac{\partial \phi_{2}}{\partial r}+\frac{1}{r} \frac{\partial^{2} \psi_{2}}{\partial \theta^{2}}+\frac{\cot \theta}{r} \frac{\partial \psi_{2}}{\partial \theta}, \\
V=\frac{1}{r} \frac{\partial \phi_{2}}{\partial \theta}-\frac{\partial^{2} \psi_{2}}{\partial r \partial \theta}-\frac{1}{r} \frac{\partial \psi_{2}}{\partial \theta} .
\end{gathered}
$$

For free harmonic vibrations, the potential functions $\phi_{1}, \phi_{2}, \psi_{1}$, and $\psi_{2}$ are expressed as follows:

$$
\begin{aligned}
& \phi_{1}=f_{1}(r) p_{l}^{m}(\cos \theta) e^{i \omega t}, \\
& \phi_{2}=f_{2}(r) p_{l}^{m}(\cos \theta) e^{i \omega t}, \\
& \psi_{1}=g_{1}(r) p_{l}^{m}(\cos \theta) e^{i \omega t}, \\
& \psi_{2}=g_{2}(r) p_{l}^{m}(\cos \theta) e^{i \omega t},
\end{aligned}
$$

where $\omega$ is the frequency of wave, $p_{l}^{m}(\cos \theta)$ is the associated Legendre polynomial, where $l$ is the order of spherical harmonic and $m=0,1, \ldots l, i$ is the complex unity, and $t$ is time. Equations (1) and (4), after a long calculation, yield

$$
\begin{aligned}
\phi_{1}= & \left(A_{1} J_{n}\left({ }_{2} \xi_{1} r\right)+B_{1} Y_{n}\left({ }_{2} \xi_{1} r\right)+A_{2} J_{n}\left({ }_{2} \xi_{2} r\right)\right. \\
& \left.+B_{2} Y_{n}\left({ }_{2} \xi_{2} r\right)\right) p_{l}^{m}(\cos \theta) e^{i \omega t}, \\
\phi_{2}= & \left(A_{1}\left({ }_{2} \delta_{1}^{2}\right) J_{n}\left({ }_{2} \xi_{1} r\right)+B_{1}\left({ }_{2} \delta_{1}^{2}\right) Y_{n}\left({ }_{2} \xi_{1} r\right)\right. \\
& \left.+A_{2}\left({ }_{2} \delta_{2}^{2}\right) J_{n}\left({ }_{2} \xi_{2} r\right)+B_{2}\left({ }_{2} \delta_{2}^{2}\right) Y_{n}\left({ }_{2} \xi_{2} r\right)\right) \\
& \times p_{l}^{m}(\cos \theta) e^{i \omega t}, \\
\psi_{1}= & \left(A_{3} J_{n}\left({ }_{2} \xi_{3} r\right)+B_{3} Y_{n}\left({ }_{2} \xi_{3} r\right)\right) p_{l}^{m}(\cos \theta) e^{i \omega t}, \\
\psi_{2}= & -\frac{{ }_{2} M_{12}}{{ }_{2} M_{22}}\left(A_{3} J_{n}\left({ }_{2} \xi_{3} r\right)+B_{3} Y_{n}\left({ }_{2} \xi_{3} r\right)\right) \\
& \times p_{l}^{m}(\cos \theta) e^{i \omega t} .
\end{aligned}
$$

In (5), $J_{n}(x), Y_{n}(x)$ are spherical Bessel functions of first and second kinds of order $n$, respectively, and

$$
\begin{aligned}
n= & -\frac{1}{2}+\frac{1}{2 p_{l}^{m}(\cos \theta)} \\
& \times\left(\left(p_{l}^{m}(\cos \theta)\right)^{2}-4 p_{l}^{m}(\cos \theta)\right. \\
& \left.\quad \times\left(p_{l}^{m^{\prime \prime}}(\cos \theta) \sin ^{2} \theta-2 p_{l}^{m^{\prime}}(\cos \theta) \sin \theta\right)\right)^{1 / 2} .
\end{aligned}
$$

The displacement components of outer part and inner part can readily be evaluated from (3) and are given by

$$
\begin{aligned}
{ }_{2} u= & \left(A_{12} D_{11}(r)+B_{12} D_{12}(r)+A_{22} D_{13}(r)\right. \\
& \left.+B_{22} D_{14}(r)+A_{32} D_{15}(r)+B_{32} D_{16}(r)\right) \\
& \times p_{l}^{m}(\cos \theta) e^{i \omega t}, \\
{ }_{2} v= & \left(A_{12} D_{21}(r)+B_{12} D_{22}(r)+A_{22} D_{23}(r)\right. \\
& \left.+B_{2}{ }_{2} D_{24}(r)+A_{32} D_{25}(r)+B_{32} D_{26}(r)\right)
\end{aligned}
$$




$$
\begin{aligned}
& \times p_{l}^{m^{\prime}}(\cos \theta) \sin \theta e^{i \omega t}, \\
{ }_{1} u= & \left(C_{1{ }_{1}} D_{11}(r)+C_{2}{ }_{1} D_{13}(r)+C_{3{ }_{1}} D_{15}(r)\right) \\
& \times p_{l}^{m}(\cos \theta) e^{i \omega t}, \\
{ }_{1} v= & \left(C_{1{ }_{1}} D_{21}(r)+C_{2}{ }_{1} D_{23}(r)+C_{3{ }_{1}} D_{25}(r)\right) \\
& \times p_{l}^{m^{\prime}}(\cos \theta) \sin \theta e^{i \omega t} .
\end{aligned}
$$

In (7),

$$
\begin{aligned}
{ }_{2} D_{11}(r)= & \frac{n}{r} J_{n}\left({ }_{2} \xi_{1} r\right)-{ }_{2} \xi_{1} J_{n+1}\left({ }_{2} \xi_{1} r\right), \\
{ }_{2} D_{12}(r)= & \frac{n}{r} Y_{n}\left({ }_{2} \xi_{1} r\right)-{ }_{2} \xi_{1} Y_{n+1}\left({ }_{2} \xi_{1} r\right), \\
{ }_{2} D_{13}(r)= & \frac{n}{r} J_{n}\left({ }_{2} \xi_{2} r\right)-{ }_{2} \xi_{2} J_{n+1}\left({ }_{2} \xi_{2} r\right), \\
{ }_{2} D_{14}(r)= & \frac{n}{r} Y_{n}\left({ }_{2} \xi_{2} r\right)-{ }_{2} \xi_{2} Y_{n+1}\left({ }_{2} \xi_{2} r\right), \\
{ }_{2} D_{15}(r)= & \left(p_{l}^{m^{\prime \prime}}(\cos \theta) \sin ^{2} \theta-(1+\cot \theta)\right. \\
& \left.\times p_{l}^{m^{\prime}}(\cos \theta) \sin \theta\right) \frac{1}{r} J_{n}\left({ }_{2} \xi_{3} r\right), \\
& \times(\cos \theta) \sin \theta) \frac{1}{r} Y_{n}\left({ }_{2} \xi_{3} r\right), \\
D_{16}(r)= & \left(p_{l}^{m^{\prime \prime}}(\cos \theta) \sin { }^{2} \theta-(1+\cot \theta) p_{l}^{m^{\prime}}\right. \\
{ }_{2} D_{21}(r)= & -\frac{1}{r} J_{n}\left({ }_{2} \xi_{1} r\right), \\
{ }_{2} D_{25}(r)= & \left(\frac{n+1}{r}\right) J_{n}\left({ }_{2} \xi_{3} r\right)-{ }_{2} \xi_{3} J_{n+1}\left({ }_{2} \xi_{3} r\right), \\
{ }_{2} D_{22}(r)= & -\frac{1}{r} Y_{n}\left({ }_{2} \xi_{1} r\right), \\
{ }_{2} D_{23}(r)= & -\frac{1}{r} J_{n}\left({ }_{2} \xi_{2} r\right), \\
r & -\frac{1}{r} Y_{n}\left({ }_{2} \xi_{2} r\right), \\
r & Y_{n}\left({ }_{2} \xi_{3} r\right)-{ }_{2} \xi_{3} Y_{n+1}\left({ }_{2} \xi_{3} r\right) .
\end{aligned}
$$

The notations ${ }_{1} D_{i j}(i=1,2 j=1,3,5)$ are the same as ${ }_{2} D_{i j}(i=1,2 j=1,3,5)$ and $\xi_{q}=$ $\left(\omega /{ }_{p} V_{q}\right)(q=1,2,3, p=1,2)$. The notations ${ }_{p} V_{1},{ }_{p} V_{2}$, and ${ }_{p} V_{3}$ are dilatational wave velocities of first and second kinds and shear wave velocity, respectively. The notations $A_{1}, B_{1}, A_{2}, B_{2}, A_{3}, B_{3}, C_{1}, C_{2}, C_{3}$ are all arbitrary constants. By substituting the displacements in (2), the relevant stresses and fluid pressure pertaining to outer and inner parts are the following:

$$
\begin{aligned}
& { }_{2} \sigma_{r r}+{ }_{2} s=\left(A_{12} M_{11}(r)+B_{12} M_{12}(r)\right. \\
& +A_{22} M_{13}(r)+B_{22} M_{14}(r) \\
& \left.+A_{32} M_{15}(r)+B_{32} M_{16}(r)\right) \\
& \times p_{l}^{m}(\cos \theta) e^{i \omega t}, \\
& { }_{2} \sigma_{r \theta}=\left(A_{12} M_{21}(r)+B_{12} M_{22}(r)\right. \\
& +A_{22} M_{23}(r)+B_{22} M_{24}(r) \\
& \left.+A_{32} M_{25}(r)+B_{32} M_{26}(r)\right) \\
& \times p_{l}^{m^{\prime}}(\cos \theta) \sin \theta e^{i \omega t}, \\
& { }_{2} s=\left(A_{12} M_{31}(r)+B_{12} M_{32}(r)\right. \\
& \left.+A_{22} M_{33}(r)+B_{22} M_{34}(r)\right) \\
& \times p_{l}^{m}(\cos \theta) e^{i \omega t}, \\
& \frac{\partial\left({ }_{2} s\right)}{\partial r}=\left(A_{12} N_{41}(r)+B_{12} N_{42}(r)\right. \\
& \left.+A_{22} N_{43}(r)+B_{22} N_{44}(r)\right) \\
& \times p_{l}^{m}(\cos \theta) e^{i \omega t}, \\
& { }_{1} \sigma_{r r}+{ }_{1} s=\left(C_{11} M_{11}(r)+C_{21} M_{13}(r)\right. \\
& \left.+C_{31} M_{15}(r)\right) p_{l}^{m}(\cos \theta) e^{i \omega t}, \\
& { }_{1} \sigma_{r \theta}=\left(C_{11} M_{21}(r)+C_{2}{ }_{1} M_{23}(r)\right. \\
& \left.+C_{31} M_{25}(r)\right) p_{l}^{m^{\prime}}(\cos \theta) \sin \theta e^{i \omega t}, \\
& { }_{1} s=\left(C_{11} M_{31}(r)+C_{2}{ }_{1} M_{33}(r)\right) p_{l}^{m}(\cos \theta) e^{i \omega t}, \\
& \frac{\partial\left({ }_{1} s\right)}{\partial r}=\left(C_{11} N_{31}(r)+C_{21} N_{33}(r)\right) p_{l}^{m}(\cos \theta) e^{i \omega t},
\end{aligned}
$$

where

$$
\begin{aligned}
{ }_{p} M_{11}(r)= & \left(\left({ }_{p} P+{ }_{p} Q\right)+\left({ }_{p} Q+{ }_{p} R\right){ }_{p} \delta_{1}^{2}\right) \\
& \times\left(\frac{n(n-1)}{r^{2}}-{ }_{p} \xi_{1}^{2}\right) J_{n}\left({ }_{p} \xi_{1} r\right) \\
& \left.+\frac{2}{{ }_{p} \xi_{1} r^{3}} J_{n+1}\left({ }_{p} \xi_{1} r\right)\right) \\
+ & \left(\left({ }_{p} A+{ }_{p} Q\right)+\left({ }_{p} Q+{ }_{p} R\right){ }_{p} \delta_{1}^{2}\right)
\end{aligned}
$$




$$
\begin{aligned}
& \times\left(\frac{2 n+L}{r^{2}} J_{n}\left({ }_{p} \xi_{1} r\right)-\frac{2{ }_{p} \xi_{1}}{r} J_{n+1}\left({ }_{p} \xi_{1} r\right)\right), \\
& { }_{p} M_{15}(r)=2{ }_{p} N\left(\frac{(n-1) L_{2}}{r^{2}} J_{n}\left({ }_{p} \xi_{3} r\right)\right. \\
& \left.-\frac{p^{p} \xi_{3} L_{2}}{r} J_{n+1}\left({ }_{p} \xi_{3} r\right)\right), \\
& { }_{p} M_{21}(r)=2{ }_{p} N\left(\frac{(1-n)}{r^{2}} J_{n}\left({ }_{p} \xi_{1} r\right)+\frac{{ }_{p} \xi_{1}}{r} J_{n+1}\right. \\
& \left.\times\left({ }_{p} \xi_{1} r\right)\right), \\
& { }_{p} M_{25}(r)={ }_{p} N\left(\left(\frac{L_{1}+n(n+1)}{r^{2}}-{ }_{p} \xi_{3}^{2}\right) J_{n}\left({ }_{p} \xi_{3} r\right)\right. \\
& \left.+\left(\frac{{ }_{p} \xi_{3}-2{ }_{p} \xi_{3}(n+1)}{r}\right) J_{n+1}\left({ }_{p} \xi_{3} r\right)\right), \\
& { }_{p} M_{31}(r)=\left({ }_{p} Q+{ }_{p} R{ }_{p} \delta_{1}^{2}\right) \\
& \times\left(\left(\frac{n(n+1)+L}{r^{2}}-{ }_{p} \xi_{1}^{2}\right) J_{n}\left({ }_{p} \xi_{1} r\right)\right. \\
& \left.+\left(\frac{2}{\xi_{p} r_{1}^{3}}-\frac{2 \xi_{p}}{r}\right) J_{n+1}\left({ }_{p} \xi_{1} r\right)\right), \\
& { }_{p} N_{41}(r)=\left({ }_{p} Q+{ }_{p} R{ }_{p} \delta_{1}^{2}\right) \\
& \times\left(\left(n^{2}+n+L\right)\right. \\
& \times\left(\frac{n}{r} J_{n}\left({ }_{p} \xi_{1} r\right)-{ }_{p} \xi_{1} J_{n+1}\left({ }_{p} \xi_{1} r\right)\right) \\
& +\left(\frac{2}{r}-(4+n){ }_{p} \xi_{1}^{2} r\right) J_{n}\left({ }_{p} \xi_{1} r\right) \\
& +\left({ }_{p} \xi_{1}^{3} r^{2}-\frac{2(n+1)}{{ }_{p} \xi_{1} r^{2}}+2{ }_{p} \xi_{1}(n+1)\right. \\
& \left.-\frac{2}{{ }_{p} \xi_{1} r^{2}}-2{ }_{p} \xi_{1}\right) \\
& \left.\times J_{n+1}\left({ }_{p} \xi_{1} r\right)\right) \text {. }
\end{aligned}
$$

In all the above, ${ }_{p} M_{12}(r),{ }_{p} M_{16}(r),{ }_{p} M_{22}(r),{ }_{p} M_{26}(r)$, ${ }_{p} M_{32}(r)$, and ${ }_{p} N_{32}(r)$ are similar expressions as in ${ }_{p} M_{11}(r)$, ${ }_{p} M_{15}(r),{ }_{p} M_{21}(r),{ }_{p} M_{25}(r),{ }_{p} M_{31}(r)$, and ${ }_{p} N_{31}(r)$ with $J_{n}, J_{n+1}$ replaced by $Y_{n}, Y_{n+1}$, respectively; ${ }_{p} M_{13}(r),{ }_{p} M_{23}(r)$, ${ }_{p} M_{33}(r)$, and ${ }_{p} N_{33}(r)$ are similar expressions to ${ }_{p} M_{11}(r)$, ${ }_{p} M_{21}(r),{ }_{p} M_{31}(r)$, and ${ }_{p} N_{31}(r)$ with ${ }_{p} \xi_{1}$ and ${ }_{p} \delta_{1}$ replaced by $\xi_{2}$ and $\delta_{2}$, respectively; ${ }_{p} M_{11}(r),{ }_{p} M_{21}(r),{ }_{p} M_{14}(r)$, ${ }_{p} M_{24}(r),{ }_{p} M_{34}(r)$, and ${ }_{p} N_{34}(r)$ are similar expressions to
${ }_{p} M_{31}(r)$ and ${ }_{p} N_{31}(r)$ with ${ }_{p} \xi_{1},{ }_{p} \delta_{1}, J_{n}$, and $J_{n+1}$ replaced by $\xi_{2}, p \delta_{2}, Y_{n}$, and $Y_{n+1}$, respectively.

In (10),

$$
\begin{aligned}
& \delta_{q}^{2}=\frac{-\left({ }_{p} P{ }_{p} R-{ }_{p} Q^{2}\right)_{1} V_{q}^{-2}}{{ }_{p} R_{p} M_{12}-{ }_{p} Q{ }_{p} M_{22}} \\
& \times\left(\frac { 1 } { r ^ { 2 } } \left(n(n+1)-r^{2} \xi_{q}^{2}\right.\right. \\
& -\left(\left(p_{l}^{m^{\prime \prime}}(\cos \theta) \sin ^{2} \theta\right.\right. \\
& \left.-2 p_{l}^{m^{\prime}}(\cos \theta) \sin \theta\right) \\
& \left.\left.\left.\times\left(p_{l}^{m}(\cos \theta)\right)^{-1}\right)\right)\right) \\
& -\frac{{ }_{p} R_{p} M_{11}-{ }_{p} Q_{p} M_{12}}{{ }_{p} R_{p} M_{12}-{ }_{p} Q_{p} M_{22}}, \\
& p=1,2, q=1,2, \\
& { }_{p} P={ }_{p} A+2{ }_{p} N \text {, } \\
& { }_{p} M_{11}={ }_{p} \rho_{11}-\frac{i_{p} b}{\omega}, \\
& { }_{p} M_{12}={ }_{p} \rho_{12}+\frac{i_{p} b}{\omega}, \\
& { }_{p} M_{22}={ }_{p} \rho_{22}-\frac{i_{p} b}{\omega}, \\
& L=\frac{1}{p_{l}^{m}(\cos \theta)}\left(p_{l}^{m^{\prime \prime}}(\cos \theta) \sin ^{2} \theta\right. \\
& \left.-2 p_{l}^{m^{\prime}}(\cos \theta) \sin \theta\right) \\
& L_{1}=\frac{1}{p_{l}^{m^{\prime}}(\cos \theta) \sin \theta} \\
& \times\left(-p_{l}^{m^{\prime \prime \prime}}(\cos \theta) \sin ^{3} \theta+p_{l}^{m^{\prime \prime}}(\cos \theta) \sin \theta\right. \\
& \left.\times(3+\sin \theta)+p_{l}^{m^{\prime}}(\cos \theta)(\sin \theta-\cos \theta)\right), \\
& L_{2}=\frac{1}{p_{l}^{m}(\cos \theta)}\left(p_{l}^{m^{\prime \prime}}(\cos \theta) \sin ^{2} \theta-(1+\cot \theta)\right. \\
& \left.\times p_{l}^{m^{\prime}}(\cos \theta) \sin \theta\right) .
\end{aligned}
$$

\section{Boundary Conditions and Frequency Equations}

The boundary conditions for the stress-free outer surface and for the perfect bonding between the outer and the inner parts are the following: 


$$
\begin{gathered}
\left({ }_{2} \sigma_{r r}+{ }_{2} s\right)-\left({ }_{1} \sigma_{r r}+{ }_{1} s\right)=0, \\
{ }_{2} \sigma_{r \theta}-{ }_{1} \sigma_{r \theta}=0, \\
{ }_{2} s=0, \\
{ }_{1} s=0, \\
{ }_{2} u-{ }_{1} u=0, \\
{ }_{2} v-{ }_{1} v=0, \\
\quad \text { at } r=r_{1}, \\
{ }_{2} \sigma_{r r}+{ }_{2} s=0, \\
{ }_{2} \sigma_{r \theta}=0, \\
{ }_{2} s=0, \\
\text { at } r=r_{2} .
\end{gathered}
$$

Equations (12) and (13) pertain to a pervious surface; in the case of impervious surface, the boundary conditions are the same as those of the pervious surface except the third and fourth equations of (12) and the third equation of (13) on fluid pressure; instead, here we have

$$
\begin{gathered}
\frac{\partial\left({ }_{2} s\right)}{\partial r}=0, \quad \frac{\partial\left({ }_{1} s\right)}{\partial r}=0 \quad \text { at } r=r_{1} . \\
\frac{\partial\left({ }_{2} s\right)}{\partial r}=0 \quad \text { at } r=r_{2} .
\end{gathered}
$$

Equations (12) and (13) result in a system of nine homogeneous equations in nine arbitrary constants: $A_{1}, B_{1}, A_{2}, B_{2}, A_{3}, B_{3}, C_{1}, C_{2}, C_{3}$. For a nontrivial solution, determinant of coefficients is zero. Accordingly, we obtain the following frequency equation for a pervious surface:

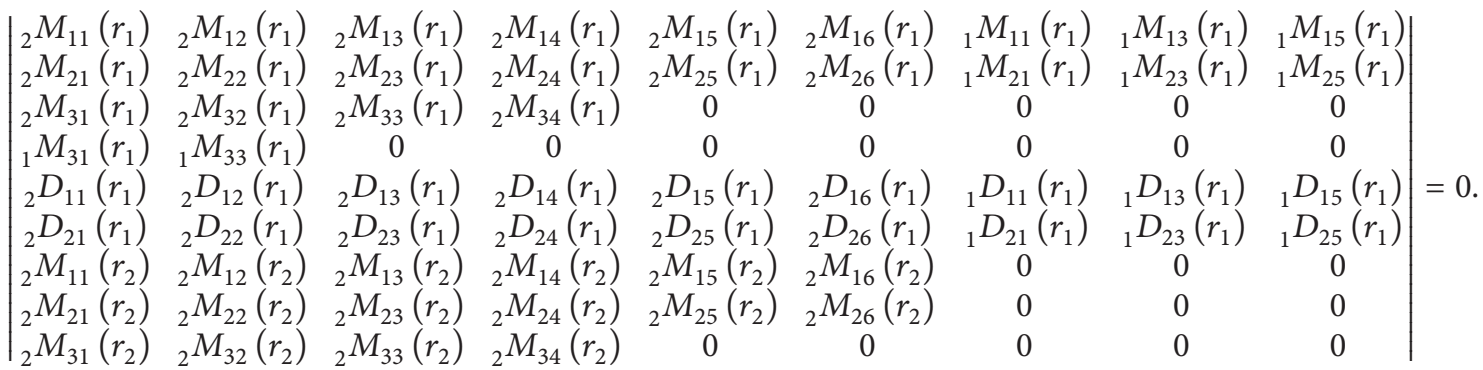

In the case of impervious surface, the frequency equation is

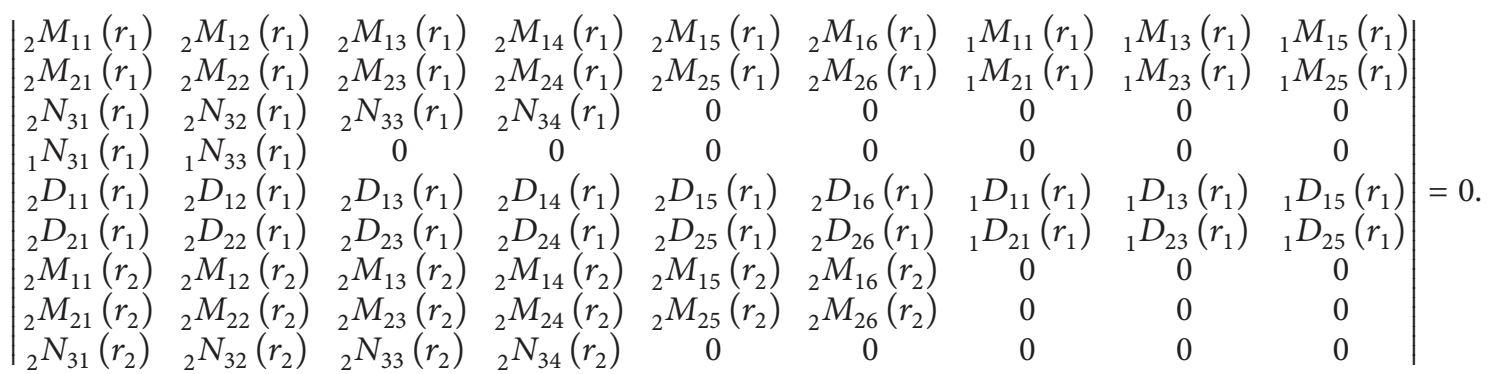

\section{Poroelastic Thick Walled Hollow Spherical Shell: A Particular Case}

The composite spherical shell will reduce to the poroelastic thick walled hollow spherical shell, under some special substitutions, that is discussed next.

Consider the case where ${ }_{2} A=A,{ }_{2} N=N,{ }_{2} Q=Q,{ }_{2} R=$ $R,{ }_{2} M_{i j}=M_{i j}$ and ${ }_{1} A=0,{ }_{1} N=0,{ }_{1} Q=0,{ }_{1} R=0$ so that ${ }_{1} M_{i j}=0$ in (16). Then, composite spherical shell will become a thick walled spherical shell in the case of pervious surface and its frequency equation is given by

$$
\left|M_{i j}\right|=0 \quad(i=1, \ldots, 6, j=1, \ldots, 6) .
$$

In (18), the elements are similar to those of (10) without left subscript. Now, we consider the case where $\xi_{i} r_{1}, \xi_{i} r_{2} \rightarrow$ $\infty \quad(i=1,2,3)$, that is, the case of $\left(h / r_{1}\right) \rightarrow 0$. In the region of small $\left(h / r_{1}\right)$, for $n \neq 0$, pertinent modes are essentially ring-extensional and ring-flexural ones [8]. Considering the determinant in (18) as a function $D$ of $\xi_{i} r_{1}(i=1,2,3)$ and $h / r_{1}$, we obtain

$$
\begin{aligned}
D\left(\xi_{i} r_{1}, \frac{h}{r_{1}}\right)= & D\left(\xi_{i} r_{1}, 0\right)+\frac{h}{r_{1}} \frac{\partial}{\partial\left(h / r_{1}\right)} D\left(\xi_{i} r_{1}, 0\right) \\
& +\frac{1}{2}\left(\frac{h}{r_{1}}\right)^{2} \frac{\partial^{2}}{\partial\left(h / r_{1}\right)^{2}} D\left(\xi_{i} r_{1}, 0\right)+\cdots
\end{aligned}
$$


It is found that

$$
D\left(\xi_{i} r_{1}, 0\right)=\frac{\partial}{\partial\left(h / r_{1}\right)} D\left(\xi_{i} r_{1}, 0\right)=0 .
$$

Hence, for a small $h / r_{1}$, we have

$$
D\left(\xi_{i} r_{1}, \frac{h}{r_{1}}\right) \approx \frac{1}{2}\left(\frac{h}{r_{1}}\right)^{2} \frac{\partial^{2}}{\partial\left(h / r_{1}\right)^{2}} D\left(\xi_{i} r_{1}, 0\right) .
$$

Further,

$$
\begin{aligned}
& \frac{\partial^{2}}{\partial\left(h / r_{1}\right)^{2}} D\left(\xi_{i} r_{1}, 0\right)
\end{aligned}
$$

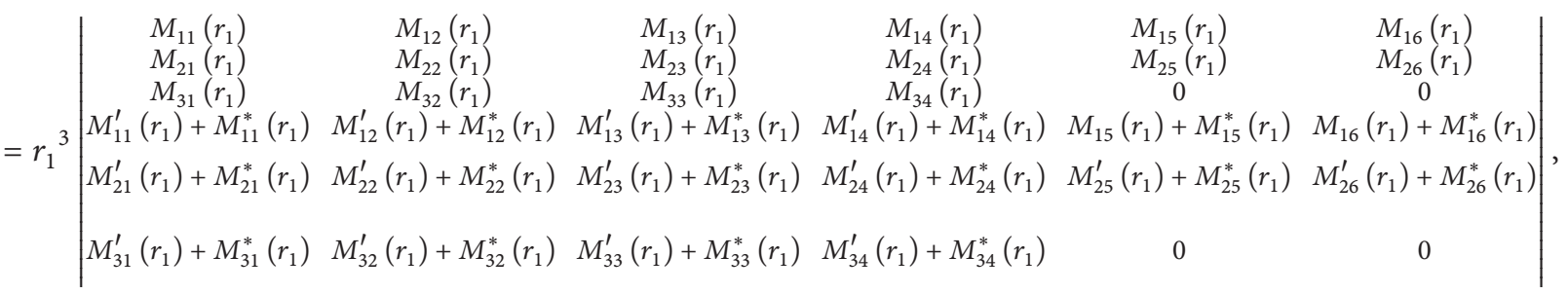

where $M_{i j}(i=1, \ldots, 6, j=1, \ldots, 6)$ are given by (18) and primes denote differentiation with respect to $r_{1}$ and $M_{i j}^{*}(i=$ $1, \ldots, 6, j=1, \ldots, 6)$ that are given in the Appendix. For a nontrivial solution, determinant of coefficient is zero, that is, $D\left(\xi_{i} r_{1}, h / r_{1}\right)=0$; accordingly, we get the frequency equation for the ring modes.

\section{Numerical Results}

Due to dissipative nature of the medium, waves are attenuated. Attenuation presents some difficulty in the definition of phase velocity. If dissipative coefficient $b$ is nonzero, then the densities will be complex numbers that make the implicit frequency equations complex valued which cannot be solved so easily. Therefore, the case $b=0$ is to be considered in what follows. Albeit the problem is poroelastic in nature, the only thing is that attenuation is not considered for the said reason. The following nondimensional parameters are introduced to investigate the frequency equations:

$$
\begin{gathered}
a_{1}=\frac{{ }_{2} P}{{ }_{1} H}, \quad a_{2}=\frac{{ }_{2} Q}{{ }_{1} H}, \quad a_{3}=\frac{{ }_{2} R}{{ }_{1} H}, \quad a_{4}=\frac{{ }_{2} N}{{ }_{1} H}, \\
d_{1}=\frac{{ }_{2} \rho_{11}}{{ }_{1} \rho}, \quad d_{2}=\frac{{ }_{2} \rho_{12}}{{ }_{1} \rho}, \quad d_{3}=\frac{{ }_{2} \rho_{22}}{{ }_{1} \rho}, \\
{ }_{2} \tilde{x}=\left(\frac{{ }_{2} V_{0}}{{ }_{2} V_{1}}\right)^{2}, \quad{ }_{1} \tilde{y}=\left(\frac{{ }_{2} V_{0}}{{ }_{2} V_{2}}\right)^{2}, \quad{ }_{1} \tilde{z}=\left(\frac{{ }_{2} V_{0}}{{ }_{2} V_{3}}\right)^{2}, \\
{ }_{1} H
\end{gathered}
$$

$$
\begin{gathered}
{ }_{1} \tilde{x}=\left(\frac{{ }_{1} V_{0}}{{ }_{1} V_{1}}\right)^{2}, \quad{ }_{1} \tilde{y}=\left(\frac{{ }_{1} V_{0}}{{ }_{1} V_{2}}\right)^{2}, \quad{ }_{1} \tilde{z}=\left(\frac{1 V_{0}}{{ }_{1} V_{3}}\right)^{2}, \\
\Omega=\frac{\omega h}{{ }_{1} c_{0}}, \quad m_{1}=\frac{c}{{ }_{1} c_{0}}, \quad c=\frac{\omega}{k} .
\end{gathered}
$$

In (23), $\Omega$ is nondimensional frequency, $c$ is phase velocity, $m_{1}$ is nondimensional phase velocity, $k$ is the wave number, and ${ }_{1} H={ }_{1} P+2{ }_{1} Q+{ }_{1} R,{ }_{1} \rho={ }_{1} \rho_{11}+2{ }_{1} \rho_{12}+{ }_{1} \rho_{22}$; also ${ }_{1} c_{0}$ and ${ }_{1} V_{0}$ are reference velocities and are given by ${ }_{1} c_{0}^{2}={ }_{1} N /{ }_{1} \rho,{ }_{1} V_{0}^{2}={ }_{1} H /{ }_{1} \rho$, and $h$ is the thickness of the poroelastic spherical shell. Let $g=r_{2} / r_{1}$, so that $h / r_{1}=$ $g-1, h / r_{2}=(g-1) / g$. Employing these nondimensional quantities in the frequency equations, we will get two implicit relations; one is between the nondimensional frequency $(\Omega)$ and the ratio of outer and inner radii $(g)$, and another is the relation between phase velocity $\left(m_{1}\right)$ and the nondimensional wave number $\left(k r_{2}\right)$. The numerical results are presented for the following cases.

5.1. Sandstone Composite Shells. Nondimensional frequency $(\Omega)$ and phase velocity $\left(m_{1}\right)$ are computed for two types of composite spherical shells, namely, composite spherical shell 1 and composite spherical shell 2 using the numerical process performed in MATLAB. In composite spherical shell 1, outer shell is made up of sandstone saturated with water [9] and inner shell is made up of sandstone saturated with kerosene [10]. In composite spherical shell 2, the roles of materials are reversed. The physical parameters of these composite spherical shells following (19) are given in Table 1. The value of $\theta$ is taken to be $30^{\circ}$ arbitrarily. The value of $m$ is taken to be 1 and the value of $l$ is taken to be 2, following [2]. The velocities of the dilatational waves and shear wave are computed using Biot's theory [4]. The numerical values are depicted in Figures 1 and 7. 
TABLE 1: Material parameters.

\begin{tabular}{lcc}
\hline $\begin{array}{l}\text { Material } \\
\text { parameters }\end{array}$ & $\begin{array}{c}\text { Composite spherical } \\
\text { shell } 1\end{array}$ & $\begin{array}{c}\text { Composite spherical } \\
\text { shell } 2\end{array}$ \\
\hline$a_{1}$ & 0.445 & 1.819 \\
$a_{2}$ & 0.034 & 0.011 \\
$a_{3}$ & 0.015 & 0.054 \\
$a_{4}$ & 0.123 & 0.780 \\
$d_{1}$ & 0.887 & 0.891 \\
$d_{2}$ & -0.001 & 0 \\
$d_{3}$ & 0.099 & 0.125 \\
${ }_{2} \tilde{x}$ & 1.863 & 0.489 \\
${ }_{2} \tilde{y}$ & 8.884 & 2.330 \\
${ }_{2} z$ & 7.183 & 1.142 \\
$b_{1}$ & 0.96 & 0.843 \\
$b_{2}$ & 0.006 & 0.065 \\
$b_{3}$ & 0.028 & 0.028 \\
$b_{4}$ & 0.412 & 0.234 \\
$g_{1}$ & 0.887 & 0.901 \\
$g_{2}$ & 0 & -0.001 \\
$g_{3}$ & 0.123 & 0.101 \\
${ }_{1} \tilde{x}$ & 0.913 & 0.999 \\
${ }_{1} \tilde{y}$ & 4.347 & 4.763 \\
${ }_{1}$ & 1.129 & 3.851 \\
\hline
\end{tabular}

Figure 1 depicts the nondimensional frequency $(\Omega)$ against the ratio of outer and inner radii $(g)$ for poroelastic composite spherical shells 1 and 2, in the case of both pervious and impervious surfaces. From the figure, it is observed that the frequency values of spherical shell 1 are, in general, less than those of shell 2 for both pervious and impervious surfaces. Also it is found that the frequency values of pervious surface are, in general, less than those of the impervious surface in the case of spherical shell 1 and greater in shell 2.

Figure 2 shows the nondimensional phase velocity $\left(m_{1}\right)$ against the nondimensional wave number $\left(k r_{2}\right)$ in the case of both pervious and impervious surfaces. The phase velocity values of spherical shell 1 are, in general, greater than those of shell 2 in the case of pervious surface and the trend is reversed in the case of impervious surface. From this figure it is also found that the phase velocity values of pervious surface are, in general, greater than those of impervious surface for both spherical shells 1 and 2 .

5.2. Spherical Bone Implanted with Titanium. If the spherical bone is implanted with titanium, then we obtain a composite spherical shell consisting of two different solids; one is bone and the other is titanium. The natural selection of titanium is obvious for its favorable characteristics including immunity to corrosion, biocompatibility, and the capacity for joining with bone, which is Osseo integration. Its density, Young's modulus, and Poisson ratio are $0.0004215 \mathrm{lb} \mathrm{sec}^{2} / \mathrm{inch}^{4}$, 105 GPA, and 0.32, respectively. Lame's constants and thereby

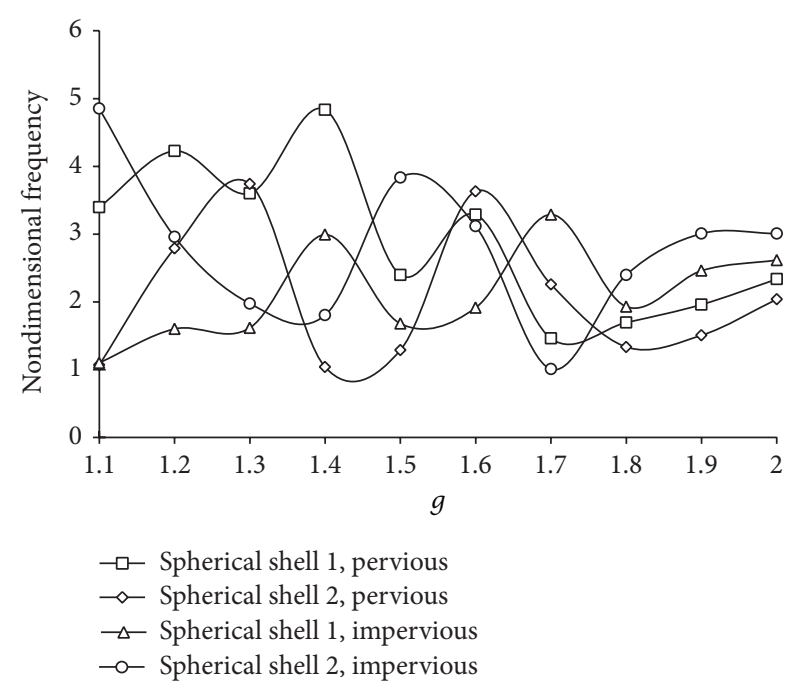

FIGURE 1: Variation of nondimensional frequency with ratio of outer and inner radii $(g)$.

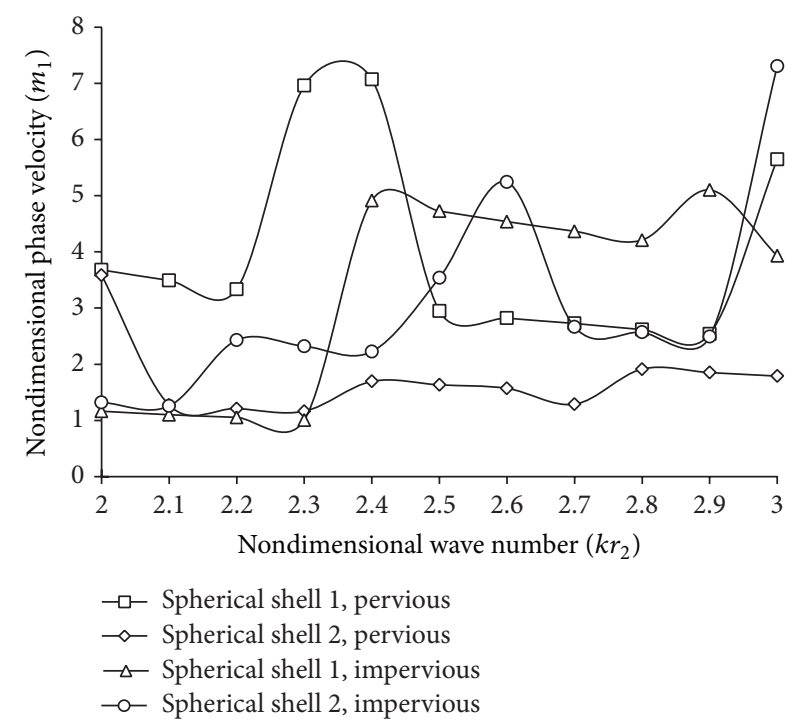

FIGURE 2: Variation of nondimensional phase velocity with wave number when $g=2$.

dilatational wave velocity and shear wave velocity are computed. The values of bone poroelastic parameters and its mass coefficients are computed by using the inputs [11]. The values of Young's modulus and Poisson ratio are taken to be $3 \times 10^{6} \mathrm{lb} /$ inch $^{2}$ and 0.28 , respectively [11]. Mass coefficients of solid part and fluid part are taken to be $1.65 \times$ $10^{-4} \mathrm{lb} \mathrm{sec}^{2} / \mathrm{inch}^{4}$ and $0.14 \times 10^{-4} \mathrm{lb} \mathrm{sec}^{2} / \mathrm{inch}^{4}$, respectively, [11]. These values are close to those of the experimental results. The values in the said study are detected at micrometer level [12]. These computations are based on the $V(z)$-curve method, which involves surface acoustic waves (SAW) that are propagating along the surface of a specimen. The dilatational wave velocities and shear wave velocity are computed, which are $V_{1}=2.016 \times 10^{5} \mathrm{inch} / \mathrm{sec}, V_{2}=$ 


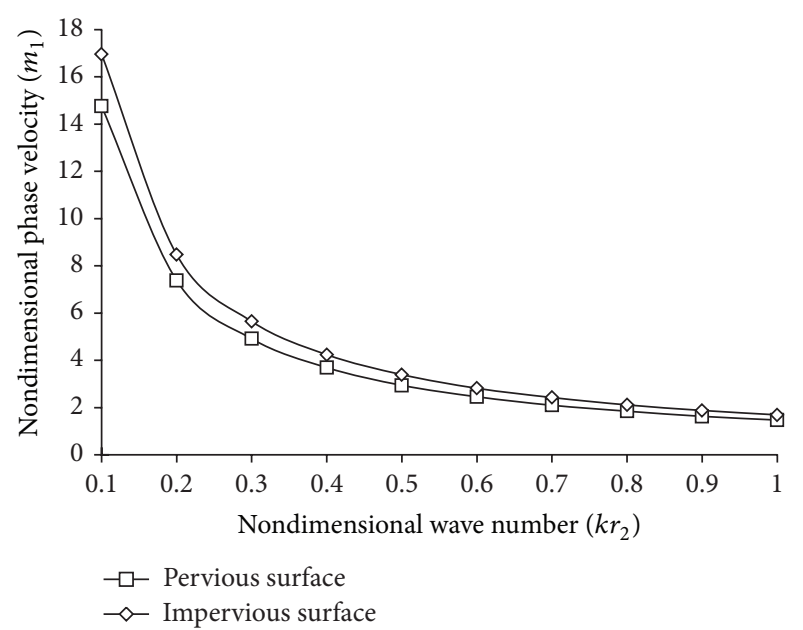

FIGURE 3: Variation of nondimensional phase velocity with wave number of spherical bone implanted with titanium for fixed $g=2$.

$1.003 \times 10^{5} \mathrm{inch} / \mathrm{sec}$, and $V_{3}=0.842 \times 10^{5} \mathrm{inch} / \mathrm{sec}$. Unlike the general case, here the second dilatational wave velocity is greater than shear wave velocity which is valid for the soft poroelastic solids [13]. Mass coupling parameter is taken to be zero [11]. The value of $g$ is fixed and is taken to be 2 . The nondimensional phase velocity $\left(m_{1}\right)$ is computed against the nondimensional wave number $\left(k r_{2}\right)$ and values are depicted in Figures 3-6. From Figure 3, it is clear that as wavenumber increases phase velocity decreases in the case of both pervious and impervious surfaces. Also, it is found that the phase velocity values of pervious surface are, in general, less than those of impervious surface.

From Figure 4, it is clear that the phase velocity values of the pervious surface are, in general, greater than those of impervious surface. There is a clear observation from Figures 3 and 4 that when the wave number lies between 0.1 and 1 , the phase velocity values decrease, and when it exceeds 1 , the nondimensional phase velocity values are periodic in the cases of both pervious and impervious surfaces.

Figures 5 and 6 depict plots of the nondimensional phase velocity $\left(m_{1}\right)$ against the nondimensional wave number $\left(k r_{2}\right)$ for $g=2,3$, and 4 in the case of both pervious and impervious surfaces. From the figures, it is clear that as $g$ increases, the phase velocity increases for both pervious and impervious surfaces.

Figure 7 depicts the nondimensional frequency $(\Omega)$ against the ratio $(g)$ for thick walled hollow spherical shells 1 and 2 in the case of ring mode. Spherical shell 1 is made up of sandstone saturated with water [9] and shell 2 is made up of sandstone saturated with kerosene [10]. From the figure, it is observed that as the ratio increases, frequency increases, and the frequency values of spherical shell 1 are, in general, greater than those of spherical shell 2. From the figures, it is clear that dispersive phenomena in the case of thick walled spherical shell and the case of its ring mode are different.

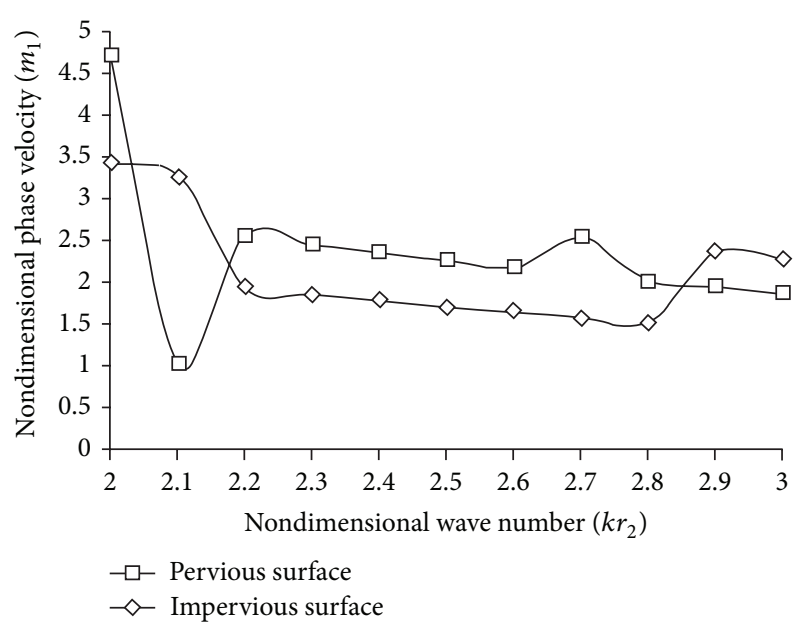

FIGURE 4: Variation of nondimensional phase velocity with wave number of spherical bone implanted with titanium for fixed $g=2$.

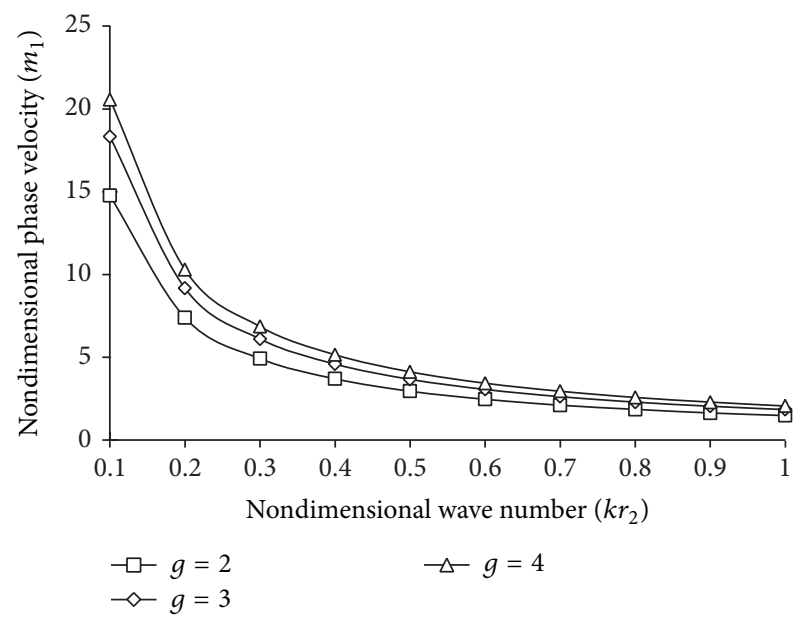

FIGURE 5: Variation of nondimensional phase velocity with wave number in spherical bone implanted with titanium for different values of $g$ in the case of pervious surface.

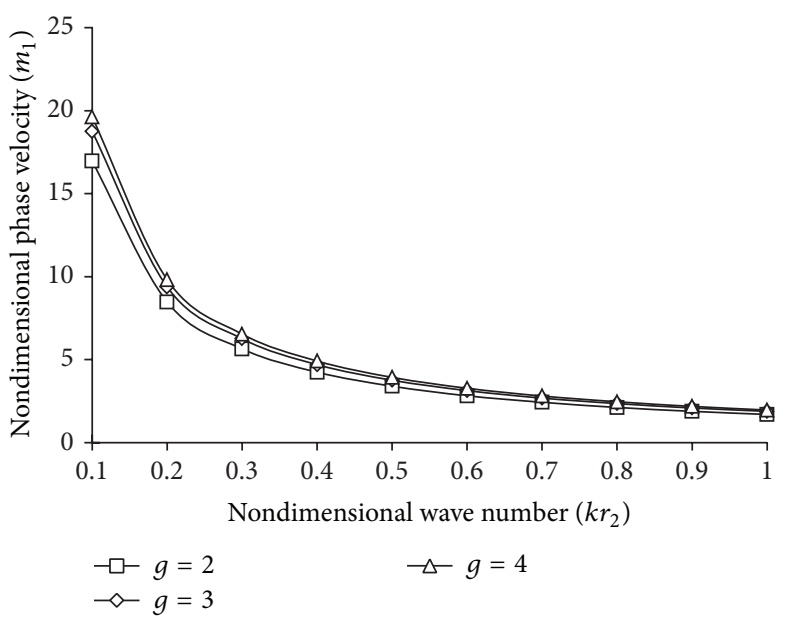

FIGURE 6: Variation of nondimensional phase velocity with wave number of spherical bone implanted with titanium for different values of $g$ in the case of impervious surface. 


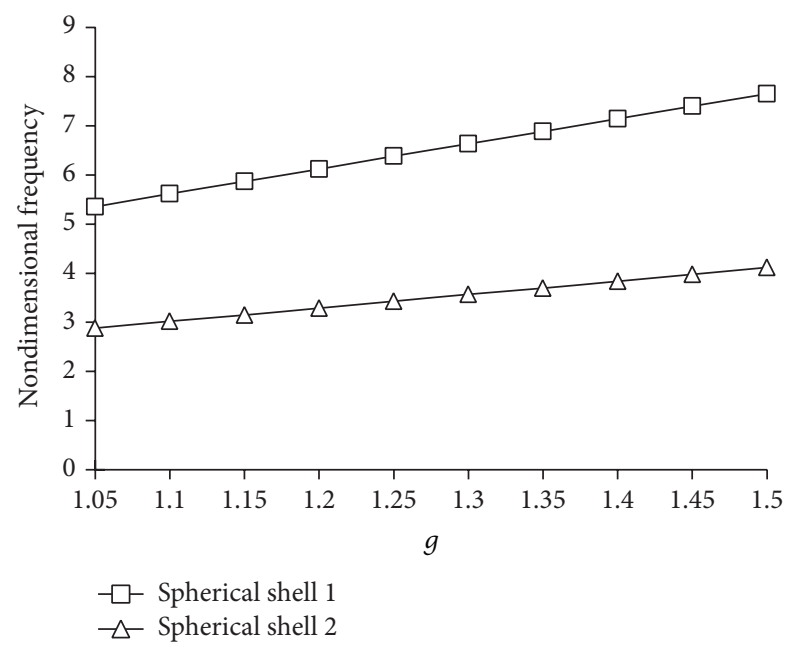

FIGURE 7: Variation of nondimensional frequency with ratio of outer and inner radii $(g)$ in the case of ring mode.

\section{Conclusion}

In the framework of Biot's theory, axially symmetric vibrations of composite poroelastic spherical shell are investigated in the case of both pervious and impervious surfaces. Two parameters, frequency and phase velocity, are investigated. Limiting cases, namely, ring modes, are studied by using appropriate approximations. From the numerical results, it is clear that dispersive behavior in the case of shell and its ring modes is distinct though both are made of the same material. Similar analysis is made for any composite spherical shell made of two different poroelastic materials if their poroelastic constants are available. This kind of analysis is useful in obtaining the unknown data in indirect way of nondestructive evaluation (NDE).

\section{Appendix}

Consider the following:

$$
\begin{aligned}
M_{11}^{*}\left(r_{1}\right)=\frac{1}{r_{1}^{3}}( & \left.(P+Q)+(Q+R) \delta_{1}^{2}\right) \\
\times & ((n(n+1)(n-2)+4 \\
& \left.+\left(\xi_{1} r_{1}\right)^{2}\left(\left(\xi_{1} r_{1}\right)^{2}-2 n^{2}-n\right)\right) \\
& \times J_{n}\left(\xi_{1} r_{1}\right)+\left(2-\frac{2 n(n+4))}{\left(\xi_{1} r_{1}\right)}\right) \\
& \left.\times J_{n+1}\left(\xi_{1} r_{1}\right)\right) \\
+ & \left((A+Q)+(Q+R) \delta_{1}^{2}\right) \\
\times & \left(\left(2 n^{3}-8 n^{2}+16 n+L\right.\right. \\
& \times\left(n^{2}-6 n+6\right)
\end{aligned}
$$

$$
\begin{aligned}
& \left.-(2 n+L-8)\left(\xi_{1} r_{1}\right)^{2}\right) \\
& \times J_{n}\left(\xi_{1} r_{1}\right)+\left(2\left(\xi_{1} r_{1}\right)^{2}\right. \\
& \left.+\left(6 L-2 n^{2}-12\right)\right) \\
& \left.\times\left(\xi_{1} r_{1}\right) J_{n+1}\left(\xi_{1} r_{1}\right)\right), \\
& M_{15}^{*}\left(r_{1}\right)=\frac{2 N L_{2}}{r_{1}^{3}}\left(\left(n^{3}-7 n^{2}+14 n-8\right)\right. \\
& \left.+(5-n)\left(\xi_{3} r_{1}\right)^{2}\right) J_{n}\left(\xi_{3} r_{1}\right) \\
& +\left(\left(\xi_{3} r_{1}\right)^{3}-\left(n^{2}+6\right)\left(\xi_{3} r_{1}\right)\right) J_{n+1}\left(\xi_{3} r_{1}\right), \\
& M_{21}^{*}\left(r_{1}\right)=\frac{2 N}{r_{1}^{3}}\left(\left(-n^{3}+7 n^{2}-2 n-4\right)+(n-5)\right. \\
& \left.\times\left(\xi_{1} r_{1}\right)^{2}\right) J_{n}\left(\xi_{1} r_{1}\right)+\left(2 n-7-\left(\xi_{1} r_{1}\right)^{2}\right) \\
& \times\left(\xi_{1} r_{1}\right) J_{n+1}\left(\xi_{1} r_{1}\right), \\
& M_{25}^{*}\left(r_{1}\right)=\frac{N}{r_{1}^{3}}\left(\left(\left(L_{1}+n^{2}+n\right)\left(n^{2}-6 n+8-\left(\xi_{3} r_{1}\right)^{2}\right)\right)\right. \\
& \left.-\left(\xi_{3} r_{1}\right)^{2}\left(L_{1}+n^{2}-8 n-2\right)\right) J_{n}\left(\xi_{3} r_{1}\right) \\
& -\left(\left(2 L_{1}+2 n^{3}+6 n^{2}+11 n+4\right)\right. \\
& \left.-\left(\xi_{3} r_{1}\right)(2 n+1)\right)\left(\xi_{3} r_{1}\right) J_{n+1}\left(\xi_{3} r_{1}\right), \\
& M_{31}^{*}\left(r_{1}\right)=\frac{(Q+R) \delta_{1}^{2}}{r_{1}^{3}} \\
& \times\left(\left(n^{2}+n+L\right)\right. \\
& \left(n^{2}-2 n-4-\left(\xi_{1} r_{1}\right)^{2}-\frac{4 n}{\left(\xi_{1} r_{1}\right)}\right) \\
& -\left(\xi_{1} r_{1}\right)^{2}\left(n^{2}-2 n-2-\left(\xi_{1} r_{1}\right)^{2}\right. \\
& \left.+2\left(\xi_{1} r_{1}\right)^{3}-16\right) J_{n}\left(\xi_{1} r_{1}\right) \\
& +\left(\left(n^{2}+n+L\right)\left(\left(\xi_{1} r_{1}\right)+4\right)+2\left(\xi_{1} r_{1}\right)^{3}\right. \\
& \times\left(\left(\xi_{1} r_{1}\right)-1\right)+\left(n^{2}+3 n+2\right) \\
& \times\left(\frac{2}{\left(\xi_{1} r_{1}\right)}-2\left(\xi_{1} r_{1}\right)^{2}-(2 n+6)\left(\xi_{1} r_{1}\right)\right. \\
& \left.\left.\left.+\frac{4(6 n+11)}{\left(\xi_{1} r_{1}\right)}\right)\right) J_{n+1}\left(\xi_{1} r_{1}\right)\right) \text {. }
\end{aligned}
$$

$M_{12}^{*}\left(r_{1}\right), \quad M_{16}^{*}\left(r_{1}\right), \quad M_{22}^{*}\left(r_{1}\right), \quad M_{26}^{*}\left(r_{1}\right), \quad$ and $\quad M_{32}^{*}\left(r_{1}\right)$ are similar expressions as in $M_{11}^{*}\left(r_{1}\right), M_{15}^{*}\left(r_{1}\right)$, $M_{21}^{*}\left(r_{1}\right), M_{25}^{*}\left(r_{1}\right)$, and $M_{31}^{*}\left(r_{1}\right)$ with $J_{n}, J_{n+1}$ replaced by 
$Y_{n}, Y_{n+1}$, respectively; $M_{13}^{*}\left(r_{1}\right), M_{23}^{*}\left(r_{1}\right)$, and $M_{33}^{*}\left(r_{1}\right)$ are similar expressions to $M_{11}^{*}\left(r_{1}\right), M_{21}^{*}\left(r_{1}\right)$, and $M_{31}^{*}\left(r_{1}\right)$ with $\xi_{1}, \delta_{1}$ replaced by $\xi_{2}, \delta_{2}$, respectively; $M_{14}^{*}\left(r_{1}\right), M_{24}^{*}\left(r_{1}\right)$, and $M_{34}^{*}\left(r_{1}\right)$ are similar expressions to $M_{11}^{*}\left(r_{1}\right), M_{21}^{*}\left(r_{1}\right)$, and $M_{31}^{*}\left(r_{1}\right)$ with $\xi_{1}, \delta_{1}, J_{n}$, and $J_{n+1}$ replaced by $\xi_{2}, \delta_{2}, Y_{n}$, and $Y_{n+1}$, respectively.

\section{Conflict of Interests}

The authors declare that there is no conflict of interests regarding the publication of this paper.

\section{References}

[1] R. Kumar, "Axially symmetric vibrations of a fluid- filled spherical shell," Acustica, vol. 21, no. 3, pp. 143-149, 1969.

[2] R. H. Rand, "Torsional vibrations of elastic prolate spheroids," Journal of the Acoustical Society of America, vol. 44, no. 3, pp. 749-751, 1968.

[3] S. Paul, "A note on the radial vibrations of a sphere of poroelastic material," Indian Journal of Pure and Applied Mathematics, vol. 7, no. 4, pp. 469-475, 1976.

[4] M. A. Biot, "The theory of propagation of elastic waves in fluid-saturated porous solid," Journal of the Acoustical Society of America, vol. 28, pp. 168-178, 1956.

[5] S. A. Shah and M. Tajuddin, "Torsional vibrations of poroelastic prolate spheroids," International Journal of Applied Mechanics and Engineering, vol. 16, pp. 521-529, 2011.

[6] S. A. Shah and M. Tajuddin, "On axially symmetric vibrations of fluid filled poroelastic spherical shells," Open Journal of Acoustics, vol. 1, pp. 15-26, 2011.

[7] B. Shanker, C. Nageswara Nath, S. Ahmed Shah, and J. Manoj Kumar, "Vibration analysis of a poroelastic composite hollow sphere," Acta Mechanica, vol. 224, no. 2, pp. 327-341, 2013.

[8] D. C. Gazis, "Exact analysis of plane-strain vibrations of thickwalled hollow cylinders," Journal of the Acoustical Society of America, vol. 30, pp. 786-794, 1957.

[9] C. H. Yew and P. N. Jogi, "Study of wave motions in fluidsaturated porous rocks," Journal of the Acoustical Society of America, vol. 60, no. 1, pp. 2-8, 1976.

[10] I. Fatt, "The Biot-Willis elastic coefficients for a sandstone," Journal of Applied Mechanics, vol. 26, pp. 296-297, 1957.

[11] J. L. Nowinski and C. F. Davis, "Propagation of longitudinal waves in circularly cylindrical bone elements," Journal of Applied Mechanics, Transactions ASME, vol. 38, no. 3, pp. 578$584,1971$.

[12] C. S. Jørgensen and T. Kundu, "Measurement of material elastic constants of trabecular bone: a micromechanical analytic study using a $1 \mathrm{GHz}$ acoustic microscope," Journal of Orthopaedic Research, vol. 20, no. 1, pp. 151-158, 2002.

[13] C.-H. Lin, V. W. Lee, and M. D. Trifunac, "On the reflection of elastic waves in a poroelastic half-space saturated with non-viscous fluid," Tech. Rep. CE 01-04, Department of Civil Engineering, University of Southern California, Berkeley, Calif, USA, 2001. 


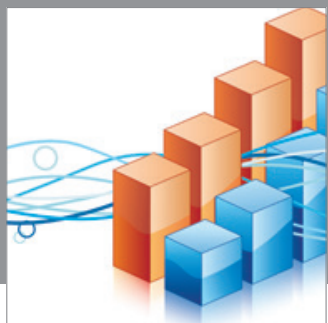

Advances in

Operations Research

mansans

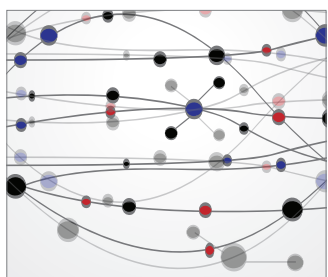

The Scientific World Journal
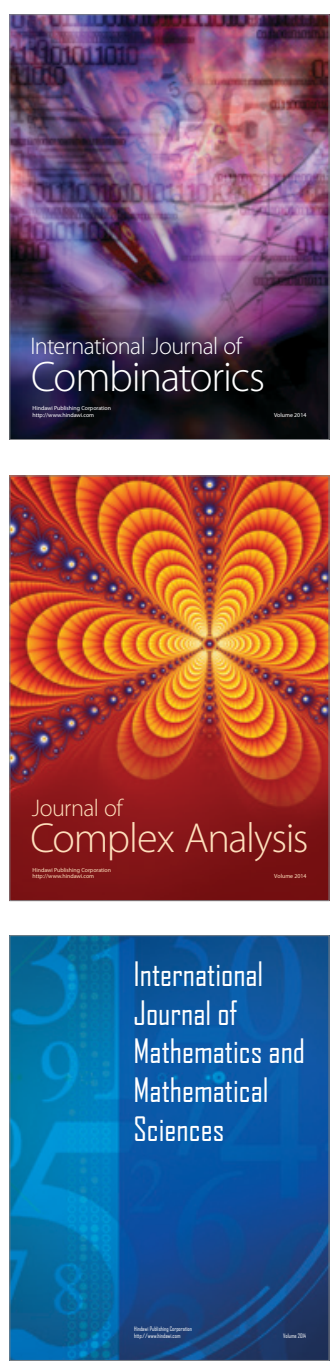
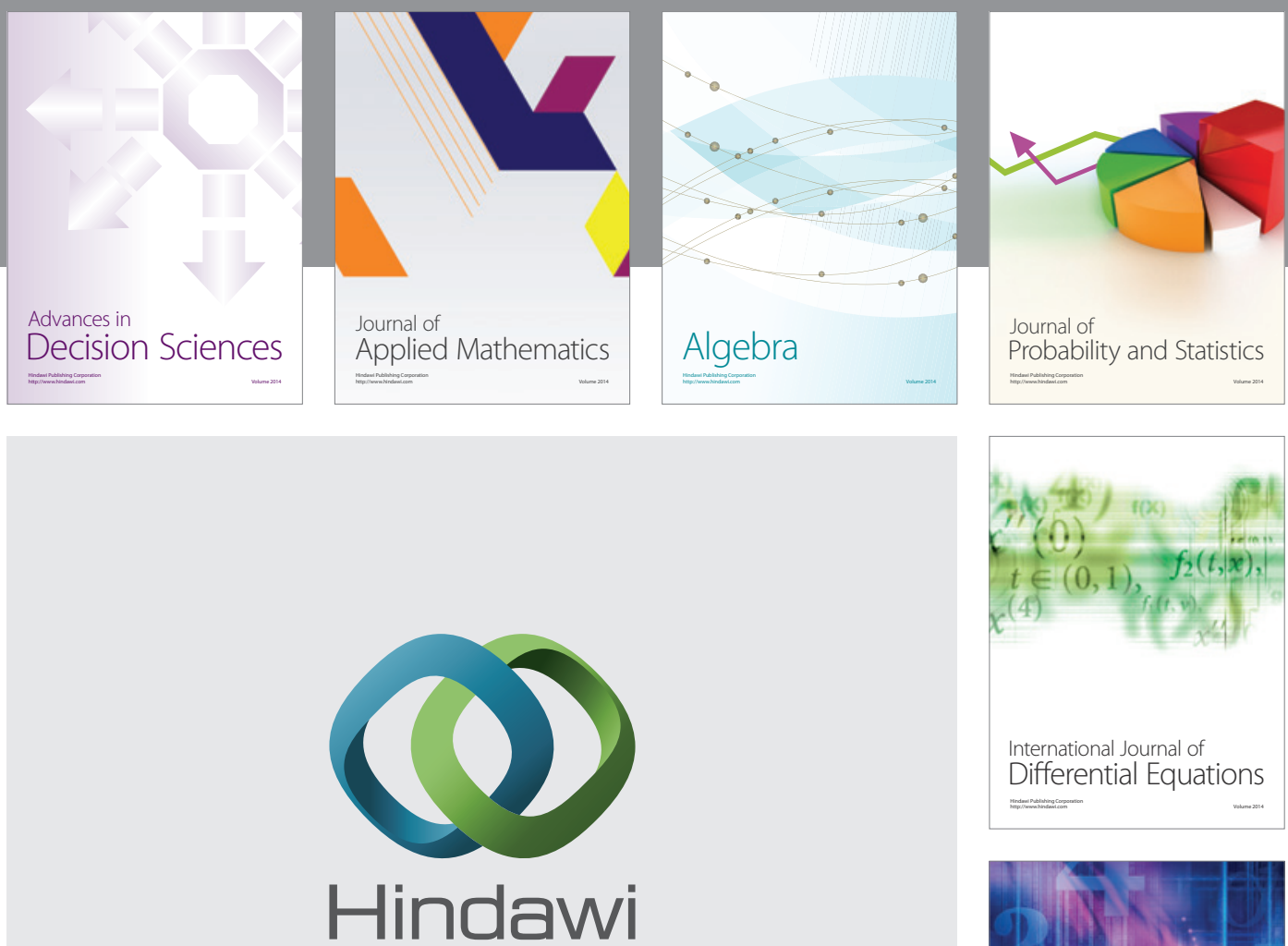

Submit your manuscripts at http://www.hindawi.com
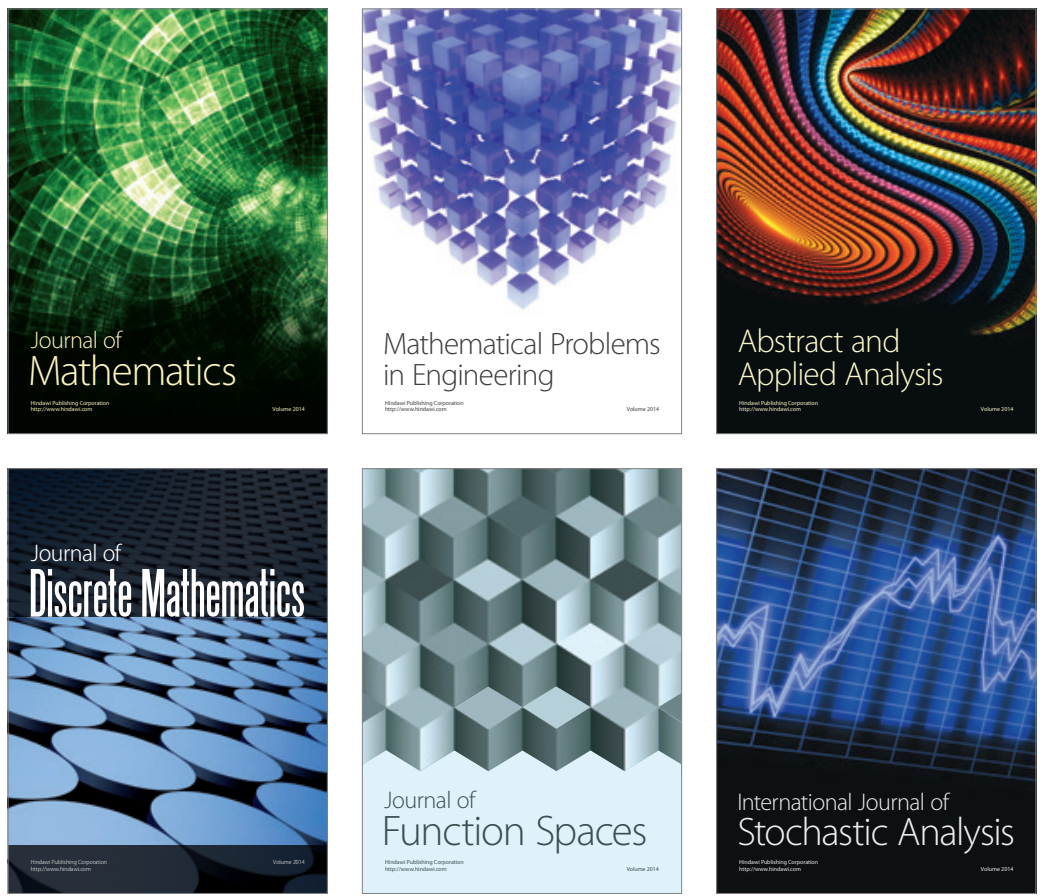

Journal of

Function Spaces

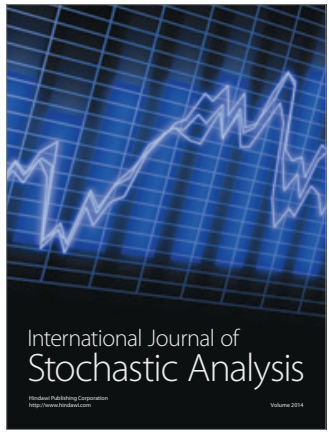

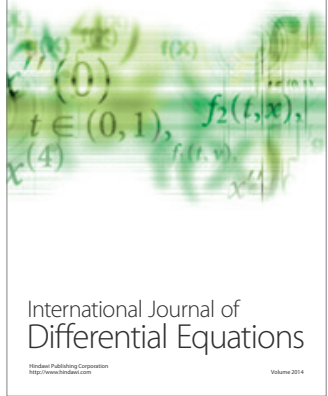
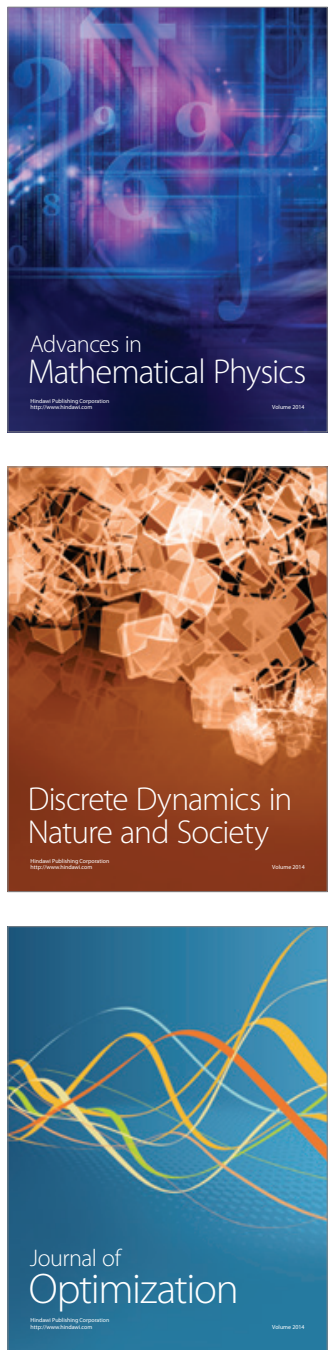